\title{
UTILIZAÇÃO DAS HEPATECTOMIAS CENTRAIS NAS CIRURGIAS HEPATOBILIARES
}

\author{
USE OF CENTRAL HEPATECTOMIES IN THE \\ HEPATOBILIARY SURGERIES
}

Fábio Luiz Waechter, TCBC-RS
José Artur Sampaio $^{1}$
Rinaldo Danesi Pinto $^{1}$
Luiz Pereira-Lima, TCBC-RS $^{2}$

\begin{abstract}
RESUMO: O tratamento das doenças hepatobiliares através de hepatectomias centrais tem sido um dos desafios técnicos mais importantes para a cirurgia neste final de século. Embora diversas técnicas tenham sido utilizadas nas últimas décadas, só recentemente estas têm sido executadas com segurança, diminuindo drasticamente as taxas de morbi-mortalidade, e com isso propiciando resultados favoráveis no tratamento das diferentes afecções hepáticas. Quer o figado apresente-se ou não com hepatopatia crônica, a integração de equipes multidisciplinares afeitas a este tipo de cirurgia e de patologia, permitiu que ressecções complexas fossem realizadas. Com o princípio de manter massa e função hepatocitária remanescente viáveis, o estudo morfológico e funcional do fígado no pré-operatório impõe que técnicas de transplante de segmentos hepáticos sejam freqüentemente utilizadas, seja na reconstrução vascular, seja na redução e conservação de massa hepática. Desta maneira, a ressecção de qualquer parte do fígado com o mínimo de utilização de derivados sangüíneos tem se mostrado factível através do conhecimento apurado da anatomia hepática e da utilização de ecografia transoperatória. Deste trabalho de revisão de diferentes técnicas de hepatectomias centrais são apresentadas, discutidas as indicações e detalhes cirúrgicos de cada uma delas.
\end{abstract}

Descritores: Hepatectomia; Neoplasia hepática.

\section{INTRODUÇÃO}

A evolução técnica da cirurgia hepática tem tornado rotineira a utilização de hepatectomias segmentares no tratamento de diversas afecções hepatobiliares, assim como durante a realização do transplante hepático. Estes novos avanços foram sendo desenvolvidos em diferentes áreas afins à cirurgia hepática, contribuindo de maneira multidisciplinar para melhora substancial dos seus resultados. Os estudos anatômicos de Couinaud ${ }^{1}$, divulgados por Bismuth ${ }^{2}$ (Figura 1), disseminaram os conhecimentos básicos da anatomia hepática, servindo estes como guia para os diversos procedimentos de ressecção hoje utilizados. Seguiu-se a formação de grupos multidisciplinares para apoio pré, trans, e pós-operatório das mesmas, destacando-se a evolução das técnicas anestésicas e dos cuidados intensivos no pós-operatório. Como corolário destas inovações, a utilização da ecografia intra-operatória tornou transparente as estruturas, outrora ocultas no interior do parênquima hepático, responsáveis por surpresas técnicas, algumas vezes catastófricas, do intra-operatório.

1. Cirurgiões do Aparelho Digestivo da FFFCMPA

2. Professor Titular de Cirurgia da FFFCMPA

Recebido em 13/9/1999

Aceito para publicação em13/6/2000

Trabalho realizado no Serviço de Cirurgia do Complexo Hospitalar Santa Casa e Fundação Faculdade Federal de Ciências Médicas de Porto Alegre - FFFCMPA. 


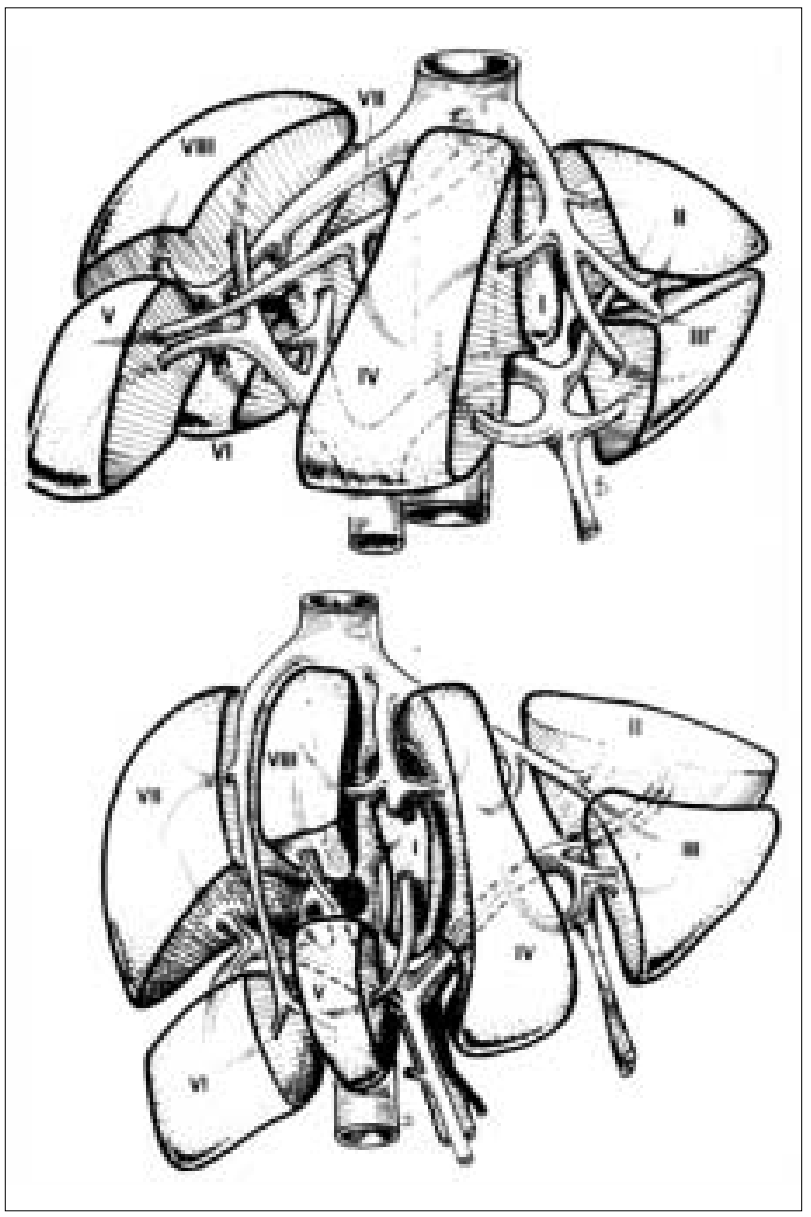

Figura 1 - Segmentação hepática preconizada por Couinaud ${ }^{1}$.

Durante o transplante, a aplicação da hepatectomia anatômica permitiu a utilização de órgãos de pacientes adultos em crianças, diminuindo os riscos de complicações advindas desta cirurgia e tornando mais ágil a passagem dos receptores pela lista de espera. Inicialmente, realizava-se a redução do fígado, quando segmentos hepáticos eram ressecados de maneira anatômica a fim de adequar o tamanho do doador com o receptor ${ }^{3}$. Posteriormente, com a carência de órgãos e o aumento da mortalidade nas filas de espera, nova era no transplante foi iniciada, utilizandose, então, a divisão do órgão doado (split) ${ }^{4,5}$, com aproveitamento da totalidade do enxerto, e de enxertos parciais de fígado, provenientes de doadores vivos ${ }^{6}$.

Da mesma maneira, a utilização rotineira de hepatectomias anatômicas para tumores hepáticos primários ou secundários conquistou seu lugar. $\mathrm{O}$ primeiro seria por razões oncológicas, quando o argumento de disseminação metastática seria realizado através de ramos portais e conseqüente disseminação para segmentos hepáticos vizinhos 7,8 e o segundo por alegação técnica, quando a ressecção anatômica diminuiria o índice de restos de parênquima desvascularizado, responsáveis por fístulas e abscessos subfrênicos no pós-operatório. Adiciona-se o fato de que tais ressecções oferecem melhor acuidade das margens de segurança adjacentes ao tumor, além de menor perda sangüínea ${ }^{9,10}$.

\section{CONSIDERAÇÕES PRÉ-OPERATÓRIAS}

Assim, o pré-operatório das exéreses anatômicas de segmentos hepáticos passou a ser obra de engenharia, planejando-se as linhas de ressecção que serão abordadas, e certificando-se da reserva funcional do parênquima remanescente. Dentro da avaliação pré-operatória do fígado, este é avaliado inicialmente quanto a sua morfologia, detalhando-se previamente a anatomia do parênquima que irá ser trabalhado. A tomografia computadorizada hepática com contraste associada ao estudo volumétrico do fígado que será ressecado e daquele que restará é de fundamental importância ${ }^{11}$. Contribuindo com este tipo de avaliação, o transplante de segmentos hepáticos tem demonstrado que, para viabilidade de sobrevida pós-operatória, é necessário cerca de $1 \%$ do peso corporal em massa hepática funcionante ${ }^{12}$. Assim, dentro da avaliação da função hepática, esta pode ser iniciada pela investigação de doenças subjacentes, freqüentemente associadas com patologias cirúrgicas do fígado, entre elas a cirrose, responsáveis por mau funcionamento do seu parênquima. Embora controversos, os métodos de avaliação funcional disponíveis vão de simples classificações, tais como a de Child e Pugh ${ }^{13,14}$, até acessos mais complexos como a excreção da bromosulftaleína ${ }^{15}$, do verde de indocianina ${ }^{16,17}$, do metabólito da lidocaína ${ }^{18}$, ou de indicadores da função metabólica tais como o índice de tolerância redox ${ }^{19}$. Desta maneira, o requinte e a ousadia das hepatectomias, combinados à baixa mortalidade, têm demonstrado que qualquer segmento hepático pode ser ressecado desde que mantenhamos massa hepática efetiva suficientemente vascularizada e drenada para cada paciente.

\section{TIPOS DE HEPATECTOMIAS CENTRAIS}

Com todas as ressecções anatômicas sendo então factíveis, associadas ao emprego rotineiro de técnicas de controle vascular, as hepatectomias por tumores passaram a ter os mais diversos aspectos conforme as devidas necessidades. Couinaud ${ }^{1}$ descreve as bases técnicas para as diversas hepatectomias anatômicas, ampliando o universo de ressecções de segmentos hepáticos restritos até então principalmente às hepatectomias direita e esquerda. Como a exérese de segmentos hepáticos laterais passou a ter menores índices de complicações, já que os pedículos dos segmentos centrais (I, IV, V, VIII) eram respeitados, as ressecções anatômicas destes últimos segmentos passaram então a ser o grande desafio técnico.

\section{Nas Neoplasias ou Estenoses Biliares Justa Hilares}

Nos casos de ressecções de neoplasias justa hilares ou, no acesso trans-hepático de estenoses hilares, algumas vezes torna-se estrategicamente necessária a ressecção anterior da porção inferior do lobo quadrado (segmento IVB). Este tipo de abordagem da confluência hilar foi primeira- 
mente realizado em 1931 por Caprio ${ }^{20}$, sendo somente repetida 30 anos após por Templeton $\mathrm{et} \mathrm{al.} .^{21}$ no tratamento da estenose iatrogênica da via biliar. No ano seguinte, Praderi et al. ${ }^{22}$, quase simultaneamente com Stephen ${ }^{23}$, difundem a ressecção do lobo quadrado ao procederem a exérese de uma lesão hilar (Figura 2). O procedimento, também utilizado por Champeau ${ }^{24}$ e Alexandre ${ }^{25}$ na França, expunha amplamente a junção dos ductos biliares, permitindo que os cotos remanescentes à ressecção fossem facilmente anastomosados e tutorados com tubos trans-hepáticos às alças jejunais em "Y de Roux".

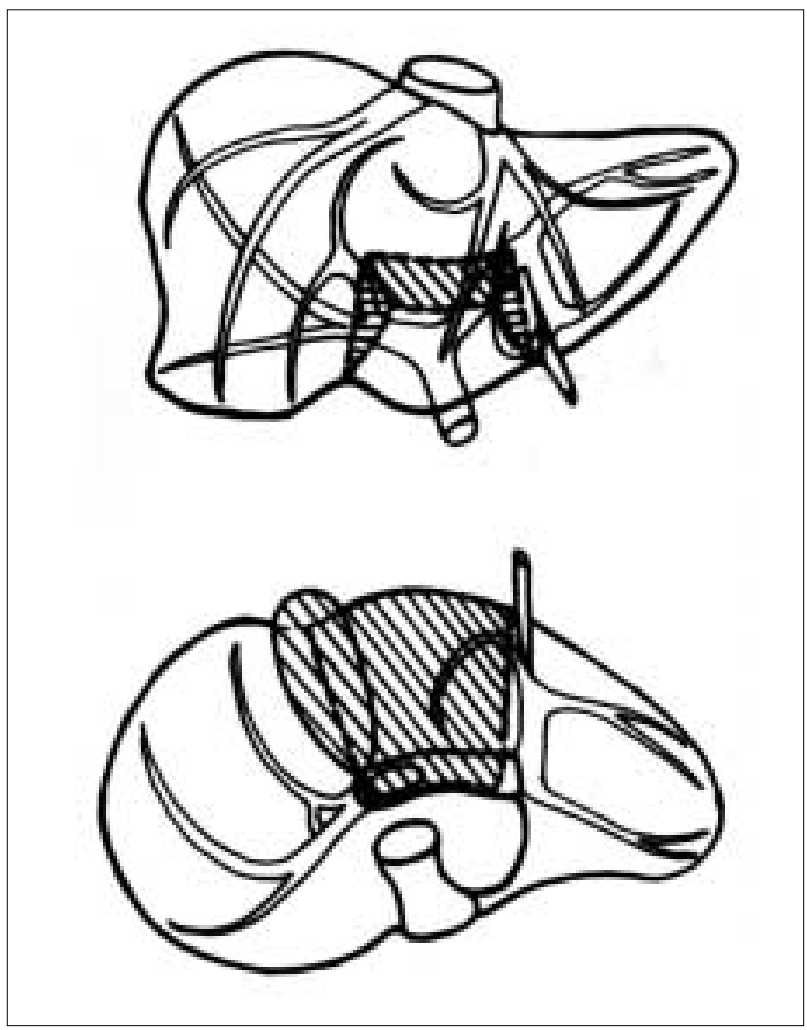

Figura 2 - Ressecção do segmento IVb preconizada por Praderi et al. ${ }^{22}$, expondo os canais biliares direito e esquerdo.

Posteriomente, Bismuth et al. ${ }^{26}$, à semelhança do procedimento descrito por Praderi, porém sem violar a placa hilar, preconizam a ressecção anatômica do segmento IVB, expondo o ducto biliar do segmento IV. Durante este procedimento, a fim de localizar o ducto biliar do segmento V, procedia-se a incisão perpendicular à fissura hepática maior, quando então ambos os ductos biliares encontrados eram anastomosados a uma única alça jejunal (Figura 3).

\section{Nos Tumores de Vesícula Biliar}

Nos casos de tumores de vesícula biliar, as ressecções hepáticas iniciaram de maneira mais singela através de Glen e Hays ${ }^{27}$, quando estes propuseram, além da colecistectomia e esqueletização do epíploo, a ressecção do leito vesicular hepático em extensão de $2 \mathrm{~cm}$ de profundidade,

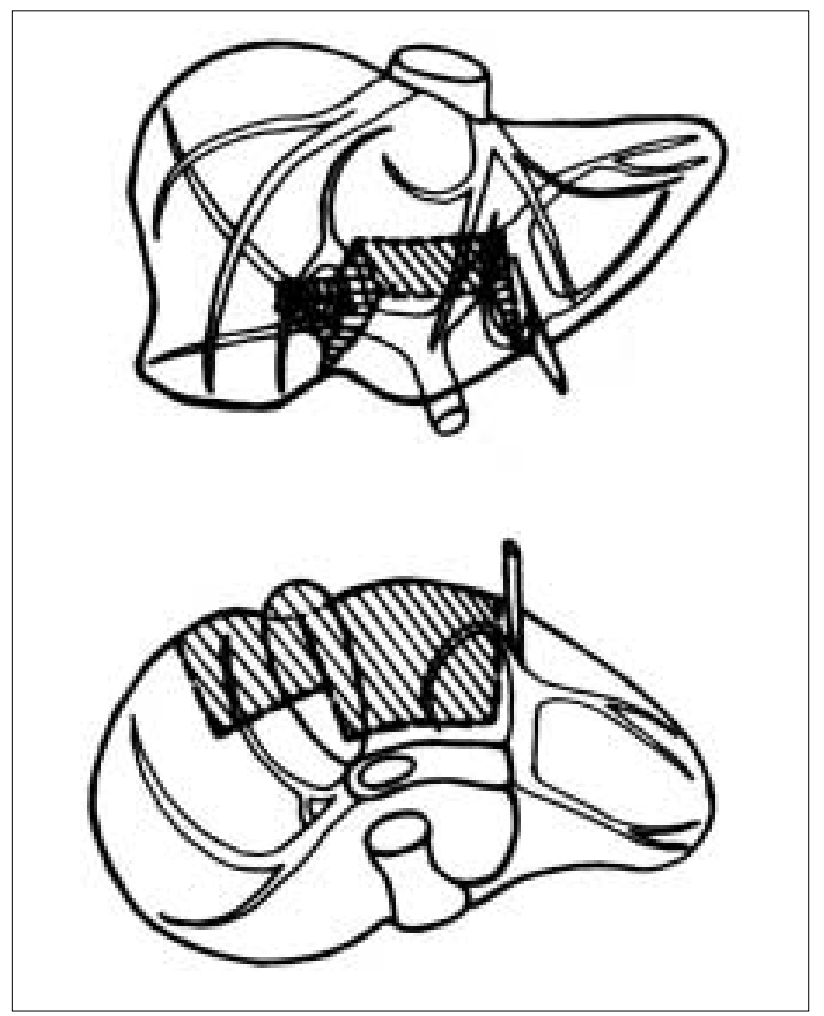

Figura 3 - Técnica empregada por Bismuth et al. ${ }^{26}$ para localizar o ducto biliar do segmento IVb e $V$.

no sentido de conter a invasão direta do fígado. Couinaud ${ }^{1}$ ao descrever a drenagem venosa da vesícula para os segmentos IV, V e VI aconselhava uma ampliação das margens de ressecção hepática para os tumores da vesícula biliar. Nesta época, Mancuso et al. ${ }^{28}$ com a mesma finalidade oncológica apregoaram a ressecção dos segmentos V e VI (Figura 4).

Preocupado com as vias de disseminação neoplásica descritas por Couinaud ${ }^{1}$, Ton That Tung ${ }^{29}$ preeconizou a trissegmentectomia dos segmentos IVb, V, VI (Figura 5).

Entretanto, a experiência daqueles que não realizavam cirurgia tão radical demonstrou que a recidiva tumoral não se fazia no segmento VI, sendo suficiente, assim, a ressecção dos segmentos IVb e V ${ }^{30}$ (Figura 6). O mapeamento mais rigoroso da drenagem venosa da vesícula biliar demonstrou posteriormente que esta era realizada principalmente para o ramo portal do segmento $\mathrm{V}$ ou para o tronco portal anterior que vasculariza os segmentos $\mathrm{V}$ e VIII ${ }^{31}$. Além disso, a constância de invasão direta dos tumores da vesícula biliar sobre o hilo hepático continuava a ser motivo de recidiva ${ }^{30}$.

Surge, assim, a hepatectomia central que consiste na ressecção dos segmentos IV, V e VIII (Figura 7). Essa intervenção descrita pela primeira vez por Pack e Miller ${ }^{32}$, foi delineada tecnicamente por Couinaud ${ }^{1}$, podendo ser realizada em planos pouco vascularizados, com a vantagem de ressecar apenas a massa hepática necessária para 


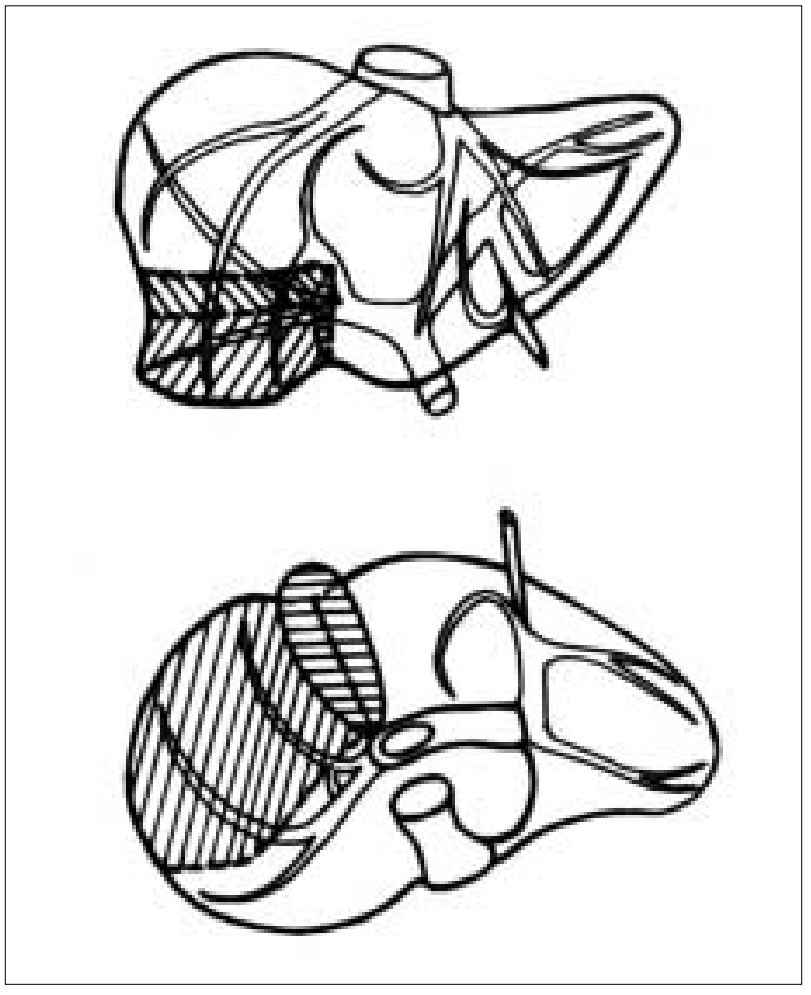

Figura 4-Exérese do segmento Ve VI descrita por Mancuso et al ${ }^{28}$.

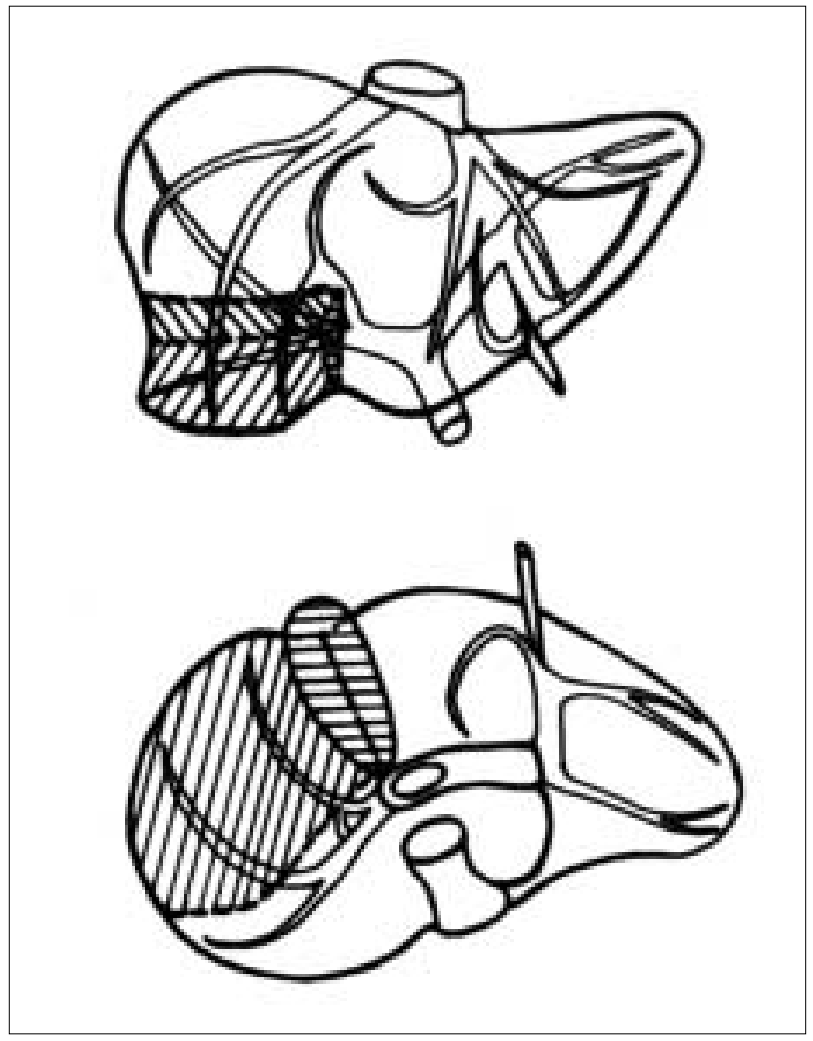

Figura 5 - Tipo de ressecção preconizado por Ton That Tung ${ }^{29}$ para o tratamento do câncer de vesícula biliar.

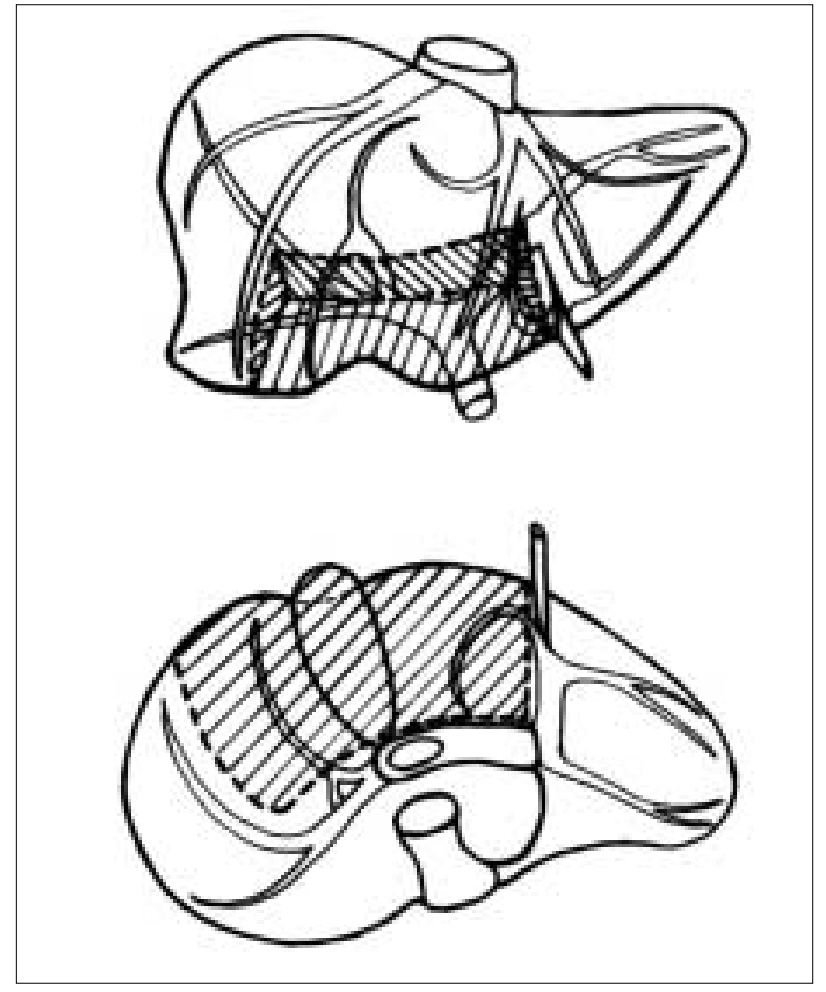

Figura 6 - Segmentectomia dos segmentos IVb e V.

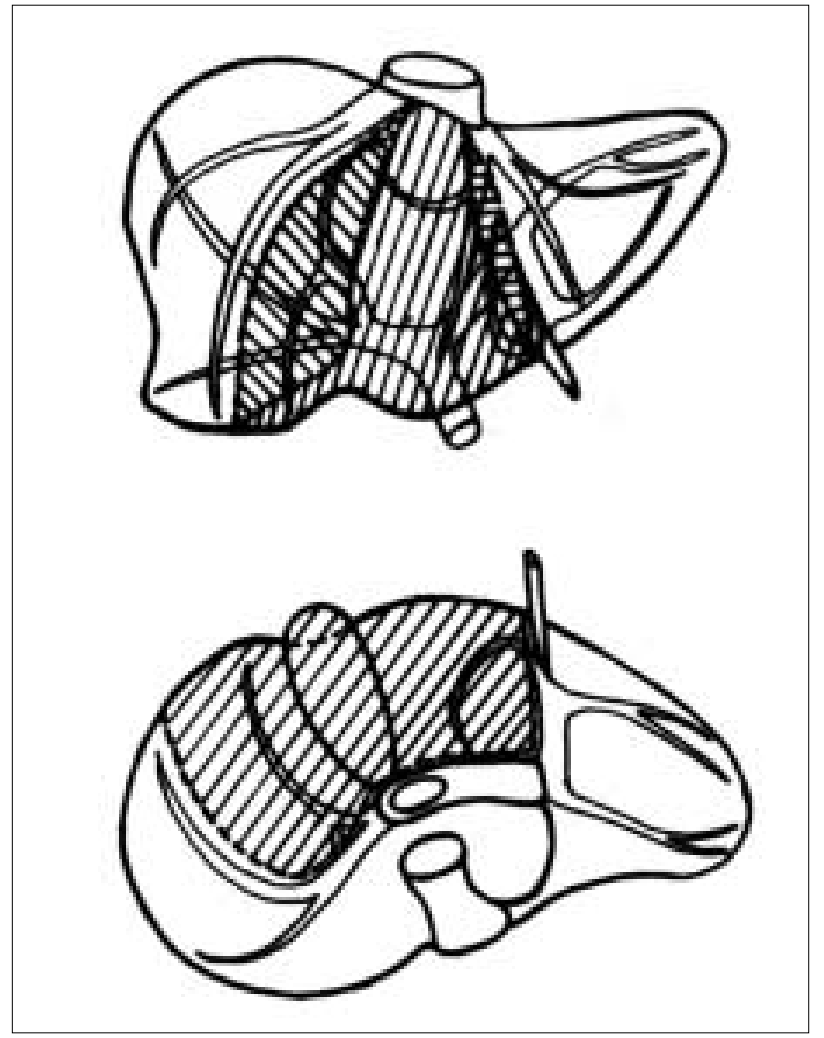

Figura 7 - Hepatectomia central apregoada no tratamento do câncer de vesícula biliar. 
proteger a recidiva, tanto local, como nos segmentos que recebem a drenagem da vesícula. Este último procedimento preserva os segmentos póstero-laterais, assim como os segmentos II e III que não estão envolvidos primariamente, assegurando, assim, volume suficiente para adequada função pós-operatória.

\section{Na Presença de Hepatopatias Crônicas}

Deve-se considerar que as cirurgias até aqui citadas são realizadas sobre parênquima com histologia residual normal, ao contrário das ressecções de carcinomas hepatocelulares ou metástases de tumores extra-hepáticos, quando, não raramente, a massa hepática restante encontra-se deficiente em sua função no começo da cirurgia. Além disso, estas lesões podem desenvolver-se em qualquer ponto do parênquima hepático, assumindo posições anatômicas que dificultam a sua retirada, ou algumas vezes impossibilitam sua exérese. Como o hepatoma invade freqüentemente ramos venosos portais com conseqüente disseminação de células tumorais ${ }^{33}$, é recomendado que qualquer ressecção do mesmo seja realizada com inclusão de todos os segmentos nutridos pelo tronco venoso portal correspondente. O mesmo não é válido para as metástases de tumores intestinais, quando a simples ressecção do mesmo com margem já é o bastante ${ }^{34}$. Desta maneira, sempre que a reserva funcional restante seja suficiente, vários segmentos hepáticos podem ser retirados concomitantemente àquele afetado macroscopicamente.

Excluindo, portanto, as ressecções dos segmentos póstero-laterais e do segmento póstero-medial que não são motivo do presente capítulo, as lesões dos segmentos mediais oferecem o que há de mais desafiador dentro da cirurgia hepática. Nos casos de tumores restritos aos segmentos V e VIII a setorectomia anterior direita (segmentos $\mathrm{V}$ e VIII) estaria indicada (Figura 8). Embora está última tenha sido idealizada por Couinaud em sua obra clássica ${ }^{1}$, a mesma já havia sido realizada um ano antes por Frédet e Bernier ${ }^{35}$ em um paciente com hemangioma. Mesmo que a década inicial desta cirurgia esteja já longínqua, poucos procedimentos deste tipo têm sido relatados na literatura $29,35,36,37$. As principais razões para isto devem-se ao fato do setor anterior direito ocupar cerca de $1 / 3$ do volume hepático total, favorecendo, assim, o desencadeamento de insuficiência do órgão. Além disso, quando o tumor assume dimensões maiores, começa a invadir segmentos adjacentes, diminuindo, assim, a efetividade oncológica de tamanha cirurgia ${ }^{37}$. Adiciona-se o fato de que as linhas de secção do parênquima são extensas, já que passam por dois dos maiores planos de segmentação.

Nos casos de tumores restritos aos segmentos III e IV, a ressecção de ambos com manutenção do segmento II pode estar indicada caso a reserva hepática esteja limitada (Figura 9). A manutenção do segmento II, sede infreqüente de disseminação dos tumores dos segmentos III e IV devido à disposição anatômica favorável do seu pedículo portal ${ }^{38}$, permite maior massa hepática residual, tão importante em uma função limítrofe. Apesar de Couinaud ${ }^{1}$ também ter sido responsável pela idealização

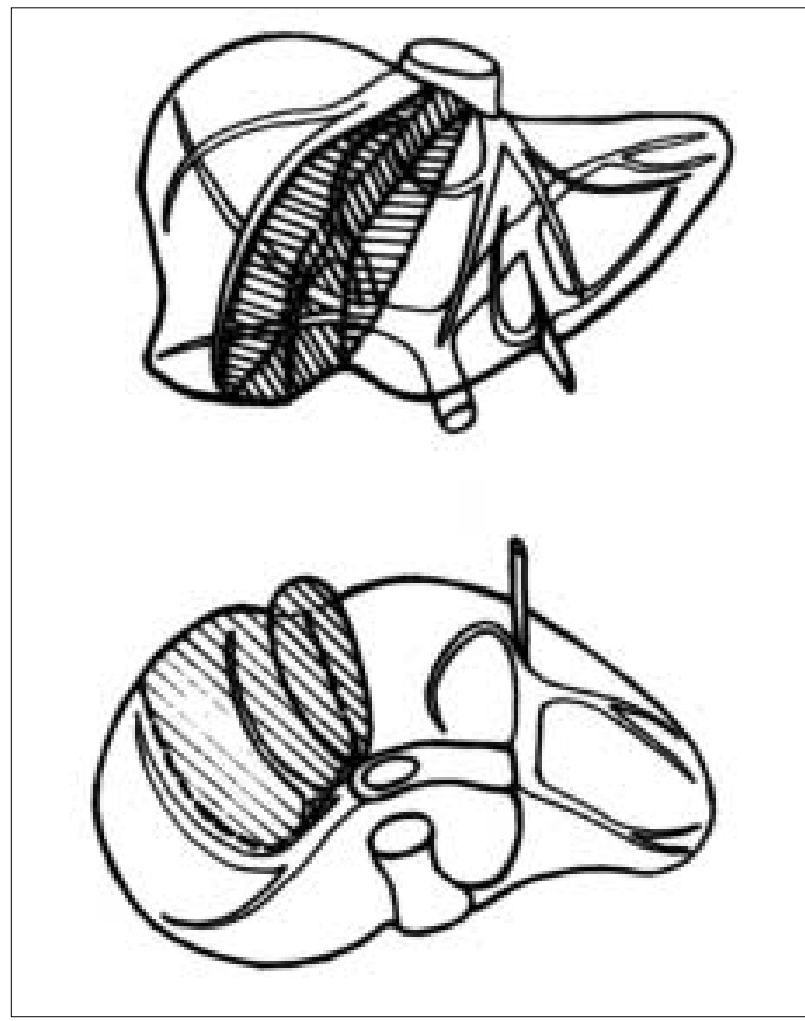

Figura 8 - Setorectomia anterior direita (segmentos V e VIII) realizada pela primeira vez por Frédet e Bernier ${ }^{35}$.

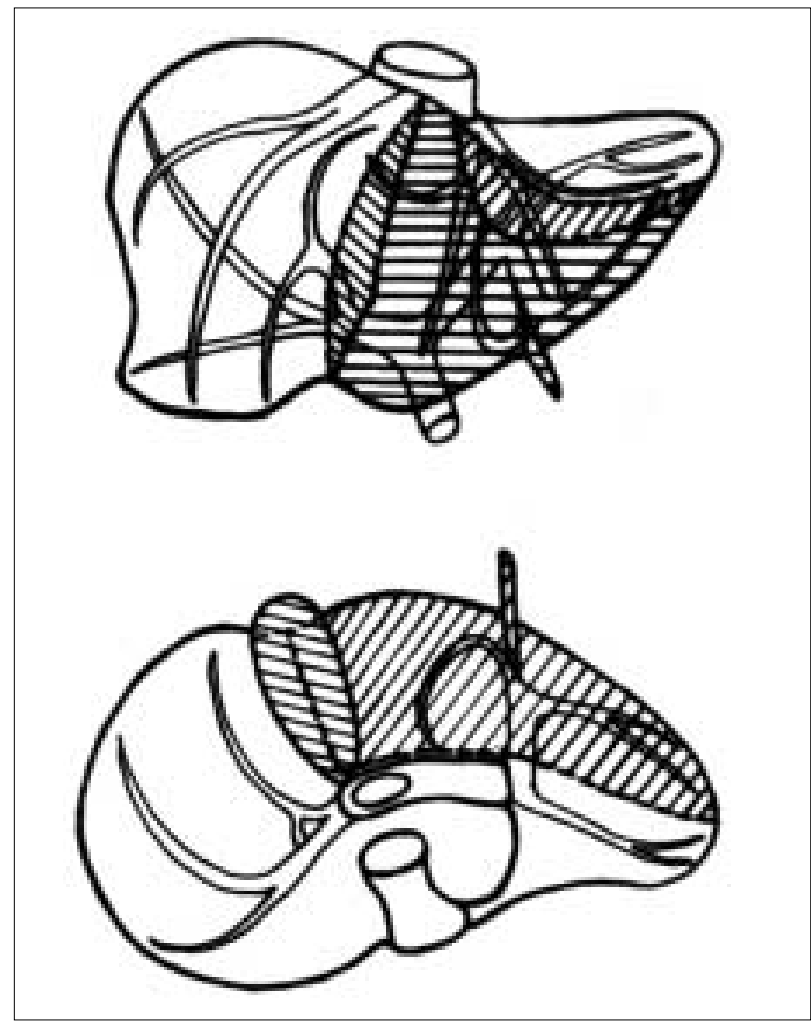

Figura 9 - Setorectomia anterior esquerda (segmentos III e IV) descrita por Kawasaki et al. ${ }^{38}$. 
de tal ressecção, coube recentemente a Kawasaki et al. ${ }^{38}$ apresentarem a primeira série de pacientes cirróticos com carcinoma hepatocelular submetidos à setorectomia anterior esquerda.

\section{Nas Lesões Próximas às Veias Hepáticas}

Situação cirúrgica extrema é aquela em que a lesão situa-se junto às veias hepáticas, acarretando risco significativo de clampeamentos prolongados ou de sangramentos intra-operatórios incoercíveis, elevando desta maneira a mortalidade cirúrgica, sobremaneira naqueles pacientes com comprometimento da função hepática, para índices proibitivos de 15 a 50\% $39,40,41$. O conhecimento da fisiologia normal do fígado permitiu apontar $90 \mathrm{minu}-$ tos como o maior tempo de isquemia normotérmica tolerável $^{42,43}$, enquanto que para fígados patológicos este tempo diminui para 60 minutos ${ }^{44}$. Dentro deste contexto, o isolamento vascular total do fígado através de clampeamentos permitiu que ressecções mais audazes fossem propostas, passando as veias hepáticas, com ou sem invasão neoplásica, a não ser mais razão para irressecabilidade. Para aqueles casos que não possuem indicação para transplante, Fortner ${ }^{45}$ idealizou pela primeira vez a exclusão vascular total do fígado associada à perfusão de solução gelada pelo parênquima para diminuir lesão de isquemia no mesmo. A evolução dos cuidados anestésicos e da cirurgia hepática permitiu que Pichlmayr et al. ${ }^{46}$ propusessem a retirada completa do fígado, perfundindo o mesmo com solução de conservação da Universidade de Wiscon$\sin { }^{47}$. Uma vez o órgão fora do organismo, era submetido a exérese da lesão tumoral, sob isquemia, em mesa separada (bench procedure), com posterior implantação do mesmo (ex situ procedure).

Kumada et al. ${ }^{48}$, tentando associar a radicalidade da exérese tumoral ex vivo em fígados cirróticos à manutenção de massa hepática suficiente, preconizam o autotransplante parcial do fígado, quando após hepatectomia parcial, a peça cirúrgica retirada é levada a outra mesa cirúrgica (Figura 10). Nesta, o fígado é perfundido com solução de conservação, a lesão tumoral é ressecada, sendo o restante então reimplantado, evitando a insuficiência hepática pósoperatória que ocorreria caso restasse apenas o segmento que manteve-se in situ.

Mesmo que os procedimentos ex situ permitam maior agressividade na exérese tumoral, estes possuem a desvantagem de ocasionar grandes áreas de dissecção, além de diversas linhas anastomóticas, sendo a maioria delas no pedículo hepático. Delrivière et al.$^{49}$ apregoam então a exclusão vascular total do fígado, associando perfusão do mesmo com solução de conservação in situ, evitando dissecção maior sobre o pedículo, assim como conseqüente diminuição do número de anastomoses no momento da implantação. Este procedimento pode (ex situ in vivo) (Figura 11) ou não (in situ in vivo) (Figura 12) ser associado à liberação das veias hepáticas da cava, entretanto sempre ligado ao pedículo hepático. Da mesma maneira da preparação ex situ, o tumor é ressecado, preservando-se os vasos que necessitam permanecer.

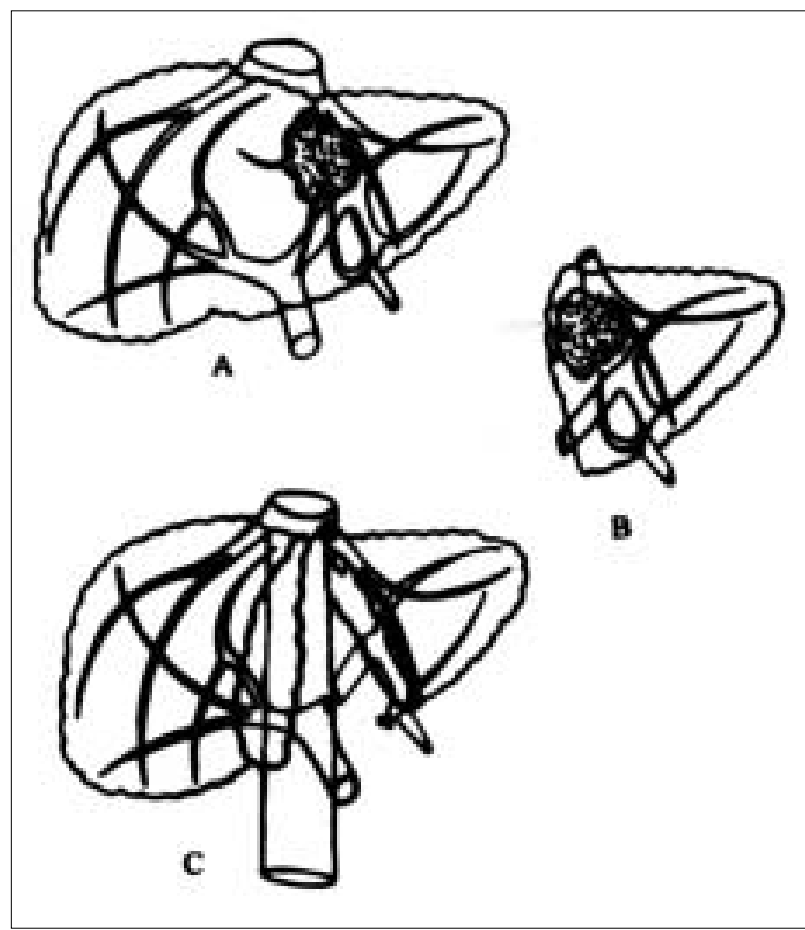

Figura 10 - Técnica preconizada por Kumada et al. ${ }^{48}$ de autotransplante parcial de fígado cirrótico, quando lesão localizada junto a veia hepática $(A)$ é ressecada após retirada de todo o lobo homolateral $(B)$, reimplantando-se este último livre de neoplasia $(C)$.

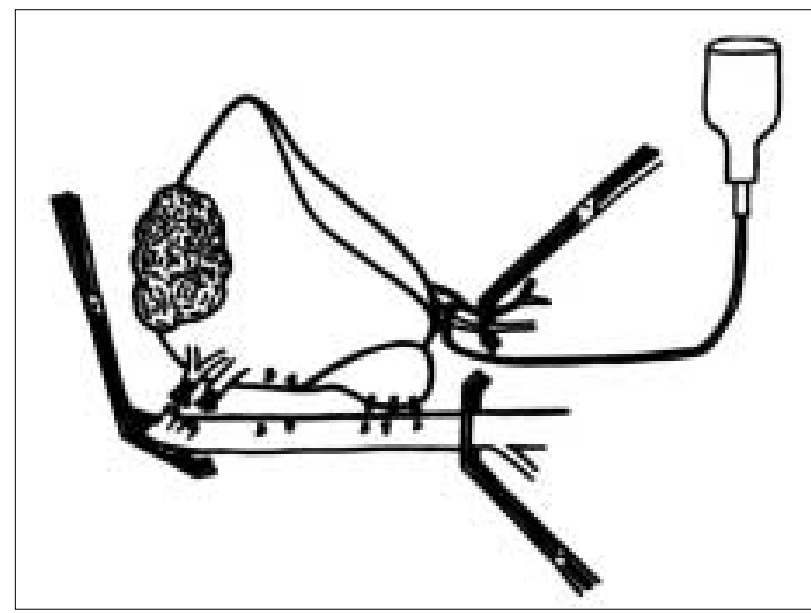

Figura 11 - Ressecção de lesão neoplásica após exclusão total do fígado, secção da veia cava supra-hepática e perfusão do mesmo com solução de conservação.

Para aqueles casos em que não é possível preservar as veias hepáticas, a evolução das técnicas de hepatectomia também permitiu a drenagem segura dos segmentos subjacentes. Estes serão restituídos através da substituição da veia hepática ressecada com enxerto venoso autólogo 50 (Figura 13), ou através da conservação de veias hepáticas acessórias inferiores suficientemente calibrosas para manter boa drenagem ${ }^{51}$ (Figura 14). 


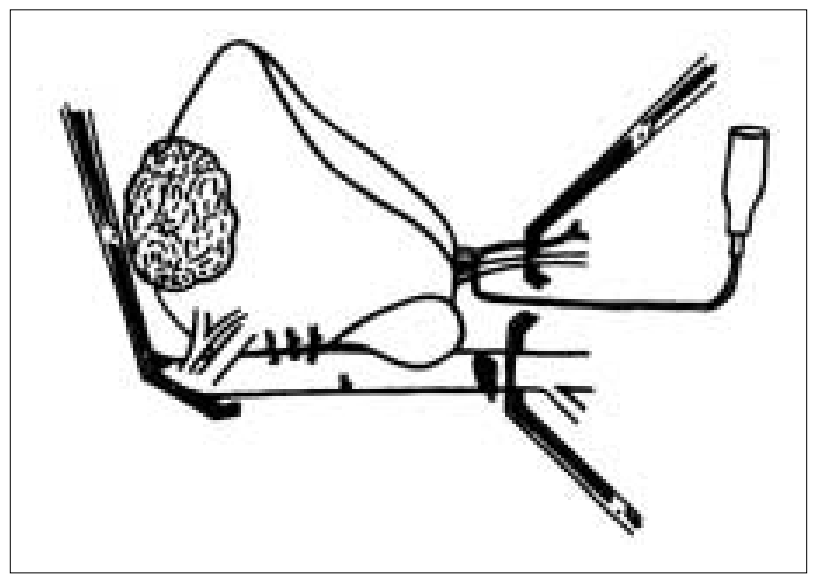

Figura 12 - Ressecção de lesão neoplásica após exclusão total do fígado e perfusão do mesmo com solução de conservação.

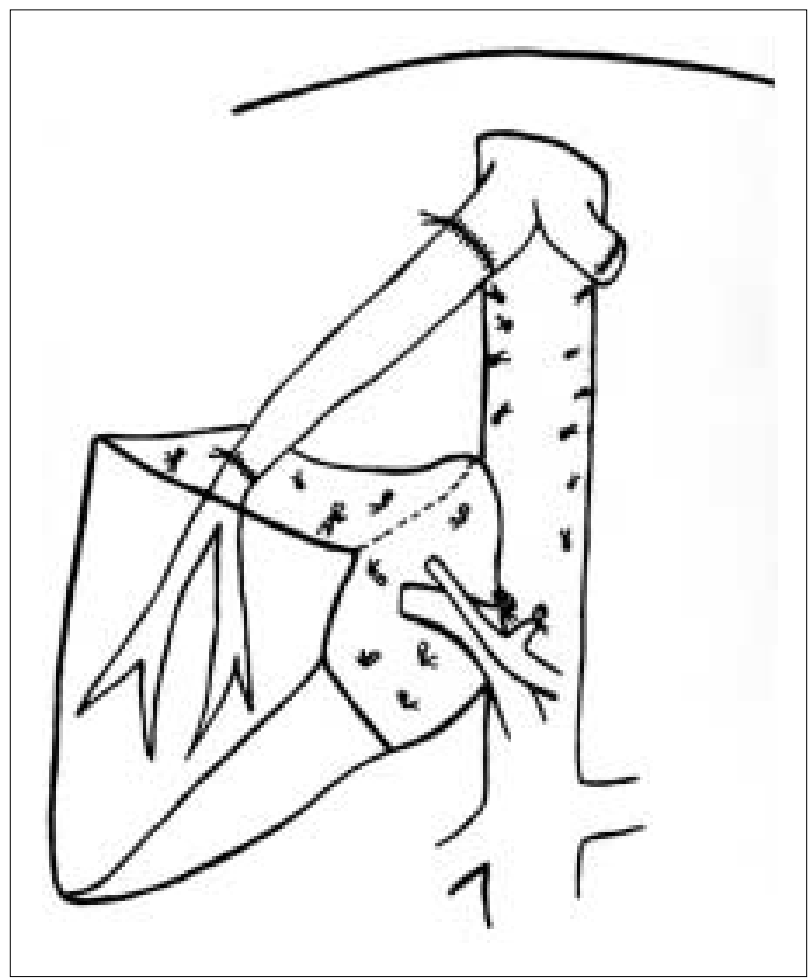

Figura 13 - Hepatectomia maior com reconstrução de veia hepática através de enxerto autólogo proposta por Takayama et al. ${ }^{50}$.

\section{Nas Lesões Localizadas no Segmento I}

Também pertencente ao grupo de ressecções centrais está a exérese das lesões do segmento I. Estas, localizando-se posteriormente ao fígado, tornam sua abordagem mais trabalhosa, não impedindo que existam diversas maneiras de proceder-se a ressecção. As abordagens mais utilizadas deste segmento consistem na exérese de um lobo inteiro do fígado ${ }^{52}$, seguida por hepatectomias anteriores ${ }^{53}$, segmentectomias anteriores ${ }^{54}$, até ressecções parciais do mesmo ${ }^{55}$ (Figura 15).

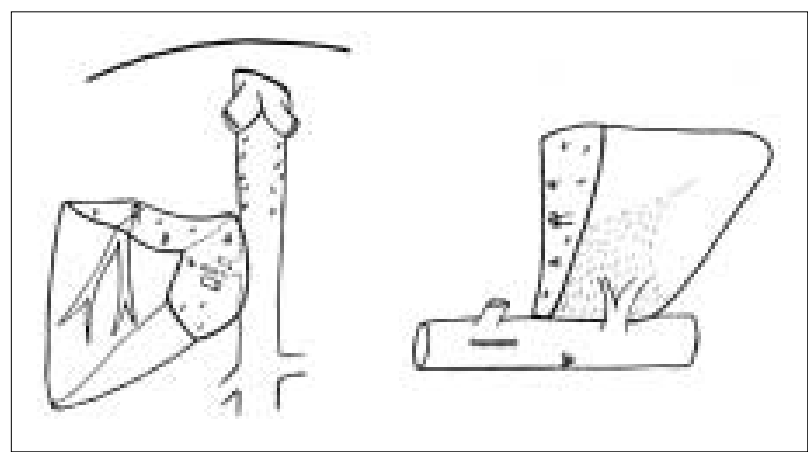

Figura 14 - Descrição de Makuuchi et al. ${ }^{51}$ para manutenção da drenagem venosa através de veias hepáticas acessórias após grande ressecção de parênquima hepático.

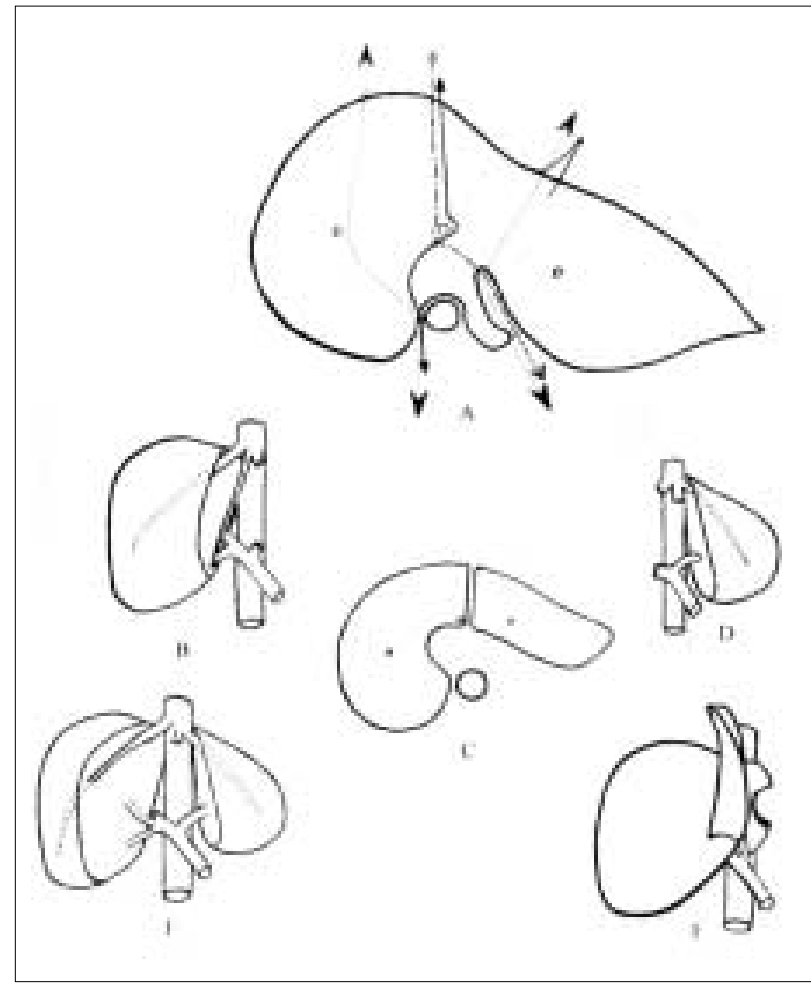

Figura 15 - Abordagens diversas para exérese de lesão localizada no segmento I (A), ressaltando-se a hepatectomia esquerda $(B)$, hepatectomia mediana $(C)$, hepatectomia direita ampliada $(D)$, hepatectomia central $(E)$ e ressecção local do lobo de Spigel $(F)$.

\section{Considerações Finais dos Autores}

Apesar da complexidade técnica das cirurgias anteriormente descritas, os princípios oncológicos de curabilidade, desafiando a ousadia e a razão do cirurgião, podem e devem ser perseguidos dentro da cirurgia hepática, com o mínimo de perdas. Se o limite das ressecções cirúrgicas dos segmentos centrais encontra-se atrelado a um serviço afeito a este tipo de cirurgia, da técnica do cirurgião responsável e, finalmente, da reserva hepatocitária que resta- 
rá, a esperança daqueles pacientes antes condenados suplica pela ousadia dos cirurgiões. A frase de Byers Shaw é própria para as ressecções aqui mencionadas:
"O homem sensato adapta-se ao mundo; aquele que é insensato persiste em tentar adaptar o mundo a ele. Assim, todo o progresso depende do homem insensato."

\begin{abstract}
The treatment of hepatobiliary diseases by central hepatectomies has been one of the most important challenges in surgical technique at the end of this century. Although different techniques were used in the last decades, only recently they have been carried out safely, drastically reducing the morbidity and mortality rates, and thus providing favorable results in treating different hepatic diseases. Whether the liver does or not present chronic liver disease, the integration of related multiple-disciplinary teams in this type of surgery and disease has allowed complex ablations, sometimes bordering on the limit of the possibility of life.Based on the principle of maintaining a viable remaining hepatocyte mass and function, the morphological and functional study of the liver during the preoperative period requires the frequent use of techniques to transplant liver segments, both for vascular reconstruction and for the reduction and conservation of the liver mass. Thus, the resection of any part of the liver with a minimum use of blood products has proved feasible with thorough knowledge of the anatomy of the liver, and the use of echography during surgery. Different central hepatectomy techniques are, thus, presented, discussing indications and surgical details of each of them.
\end{abstract}

Key Words: Hepatectomy; Hepatic neoplasm.

\title{
REFERÊNCIAS
}

1. Couinaud C - Le foie: Etudes anatomiques et chirurgicales. New York, NY. Masson Publishers, 1957.

2. Bismuth H - Surgical anatomy and anatomical surgery of the liver. World J Surg 1982; 6: 3-9.

3. Bismuth H, Houssin D - Reduced-size orthotopic liver graft in hepatic transplantation in children. Surgery 1984; 95: 367-370.

4. Pichlmayr R, Ringe B, Gubernatis G, et al. - Transplantation einer Spenderleber auf bei zwei Empfänger (splitting-transplantation). Eine neue Method in der Weiterent-wickling der Lebersegmenttransplantation. Langenbecks Arch Chir 1988; 373: 127-130.

5. Bismuth H, Morino M, Castaing D - Emergency orthotopic liver transplantation in two patients using one donor liver. Br J Surg 1989;76: 722-724.

6. Raya S, Nery JR, Mies S - Liver transplantation from live donors. Lancet 1989; 2: 497-498.

7. Kanemazsu T, Takenaka K, Matsumata T - Limited hepatic resection for selected cirrhotic patients with primary liver cancer. Ann Surg 1984; 199: 51-54.

8. Castaing D, Garden J, Bismuth H - Segmental liver resection using ultrasound-guided selective portal venous occlusion. Ann Surg 1989; 210: 20-23.

9. Bismuth H, Houssin D, Castaing D - Major and minor segmentectomies "réglées"in liver surgery. World J Surg 1982; 6: 10-24.

10. Bismuth H, Houssin D, Mazmanian G - Postoperative liver insufficiency: prevention and management. World $J$ Surg 1983; 7: 505-510.

11. Kahn CE Jr,Bae KT, Giger ML, Chen CT - "Three-dimensional visualization of the liver from computed-tomography data". In: Proceedings of the first conference on visualization in biomedical computing. Los Alamitos, CA: IEEE Computer Society Press, 1990, pp 447-449.
12. Lo CM, Fan ST, Chan JFK, et al. - Minimum graft volume for successful adult-to-adult living donor liver transplantation for fulminant hepatic failure. Transplantation 1996; 62: 696-698.

13. Child CG. The liver and portal hypertension. WB Saunders Co, Philadelphia, 1954. Pág.: 50.

14. Pugh RNH, Murray-Lyon IM, Dawson JL - Transection of the esophagus for bleeding esophageal varices. $\mathrm{Br} \mathrm{J}$ Surg 1973; 60: 646-649.

15. McIntosh EL, Minuk GY - Hepatic resection in patients with cirrhosis and hepatocellular carcinoma. Surg Gynecol Obstet 1992; 174:245-254.

16. Gottlieb ME, Stratton HH, Newell JC, et al. - Indocyanine green: its use as an early indicator of hepatic dysfunction following injury in man. Arch Surg 1984; 119: 264-268.

17. Matsumata T, Kanematsu T, Yoshida Y - The indocyanine green test enables prediction of postoperative complications after hepatic resection. World J Surg 1987; 11: 678-681.

18. Bruzzone P, Alfani D, Rossi M, et al. - Monoethylglycinexylidide clearance test: Its role as a part of brain-dead donors' evaluation for liver transplantation. Transplantation Proceedings 1993; 25: 3112-3113.

19. Mori K, Ozawa K, Yamamoto Y - Response of hepatic mitochondrial redox tolerance test as a new prediction of surgical risk in hepatectomy. Ann Surg 1990; 211: 438-446.

20. Caprio G - Un caso de extirpacion del lobulo izquierdo del higado. Bull Soc Cir Urug Montevideo 1931; 2:159.

21. Templeton J, Dodd GD - Anatomical separation of the right and left lobes of the liver for intrahepatic anastomosis of the biliary ducts. Ann Surg 1963; 157: 287. 
22. Praderi R, Parodi H, Delgado B - Tratamiento de las obstrucciones neoplásicas de la vía biliar suprapancreática. An Fac Med Montevideo 1964; 49: 221.

23. Stephen JL - Quadrate lobectomy. Proc R Soc Med 1964; 57: 551.

24. Champeau M - La segmentectomie IV dans l'exposition chirurgicale des voies biliaires intra-hépatiques. Mem Acad Chir 1967; 93: 164.

25. Alexandre $\mathrm{JH}$, Chambon $\mathrm{H}$, Poilleux $\mathrm{F}$ - La résection dans les cancers des canaux biliaires intrahépatiques intéressant la convergence. Ann Chir 1975; 29: 8.

26. Bismuth H, Lechaux JP - "Les anastomoses biliodigestives intrahépatiques". In: Encyclopédie Médico-Chirurgicale. Editions Techniques. Paris: 1970.

27. Glen F, Hays D - The scope of radical surgery in the treatment of malignant tumores of the extrahepatic biliary tract. Surg Gynecol Obstet 1954; 99: 529-541.

28. Mancuso M, Natalini E, Del Grande G - Contributo alla conoscenza della struttura segmentaria del fegato in rapporto al problema della resezione epatica. Policlinico Sez Chir 1955; 72.

29. Tung TT - Les résections majeures et mineures du foie. Paris, Masson, 1979.

30. Bismuth H, Malt R - Carcinoma of the biliary tract. N Engl J Med 1979; 301: 704.

31. Gall P, Kockerling F, Scheele J - Radical operations for carcinoma of the gallbladder: present status in Germany. Word J Surg 1991; 15: 328-336.

32. Pack G, Miller T - Middle hepatic lobectomy for cancer. Cancer 1961; 141: 295-298.

33. Ozawa K, Takayasu T, Kumada K, et al. - Experience with 225 hepatic resections for hepatocellular carcinoma over a 4 year period. Am J Surg 1991; 161: 677-682.

34. Nordlinger B, Jaeck D - Traitment des métastases hépatiques des cancers colorectaux. Springer-Verlag Paris, 1992.

35. Frédet M, Bernier E - Hépatectomie segmentaire droite réglée pour volumineux hémangiome caverneus de foie. Mém Acad Chir 1956; 82: 1060-1062.

36. McBride CM, Wallace S - Cancer of the right lobe of the liver: a variety of operative procedures. Arch Surg 1972; 105: 289-296.

37. Makuuchi M, Hashikura Y, Kawasaki S, et al. - Personal experience of right anterior segmentectomy(segments V and VIII) for hepatic malignancies. Surgery 1993; 114: 52-58.

38. Kawasaki S, Makuuchi M, Harada H, et al. - A new alternative hepatectomy method for resection of segments 3 and 4 of the liver. Surg Gynecol Obstet 1992; 175: 267-269.

39. Bismuth H, Houssin D, Ornowski J - Liver resections in cirrhotic patients: a western experience. World J Surg 1986; 10: 311-317.

40. Nagasue N, Kohno H, Chang YC - Liver resection for hepatocellular carcinoma. Results of 229 consecutive patients during 11 years. Ann Surg 1993; 217: 375384.

41. Thompson HH, Tompkins RK, Longmire WP - Major resections. A 25 year experience. Ann Surg 1983; 197: 375-388.

42. Huguet C, Gavelli A, Chieco A - Liver ischemia for hepatic resection: where is the limit? Surgery 1992; 111: 251259.
43. Hannoun L, Borie D, Delva E - Liver resection with normothermic ischemia exceeding 1 hour. Br J Surg 1993; 80: 1161-1165.

44. Yamaoka Y, Ozawa K Kumada K - Total vascular exclusion for hepatic resections in cirrhotic patients: application of venovenous bypass. Arch Surg 1992; 127: 276280.

45. Fortner JG, Shiu MH, Kinne DW - Major hepatic resection using vascular isolation and hypotermic perfusion. Ann Surg 1974; 180: 644-652.

46. Pichlmayr R, Grosse H, Hauss J - Technique and preliminary results of extracorporeal liver surgery(bench procedure) and of surgery on the in sith perfused liver. Br J Surg 1990; 77: 21-26.

47. Belzer FO, Southard JH - Principles of solid organ preservation by cold storage. Transplantation 1988: 45: 673676.

48. Kumada K, Yamaoka Y, Morimoto T, et al. - Partial autotransplantation of the liver in hepatocellular carcinoma complicating cirrhosis. Br J Surg 1992; 79: 566567.

49. Delrivière L, Hannoun L - In situ and ex situ in vivo procedures for complex major liver resections requiring prolonged hepatic vascular exclusion in normal and diseased livers. J Am Col Surg 1995; 181: 272-276.

50. Takayama T, Nakatsuka T, Yamamoto J, et al. - Re-reconstruction of a single remnant hepatic vein. Br J Surg 1996; 83: 762-763.

51. Makuuchi M, Hasegawa H, Yamazaki S, et al. - Four new hepatectomy procedures for resection of the right hepatic vein and preservation of the inferior right hepatic vein. Surg Gynecol Obstet 1987; 164: 68-72.

52. Iwasaki Y, Okamura T, Ozaki A - Surgical treatment for carcinoma at the confluence of the major hepatic ducts. Surg Gynecol Obstet 1986; 162: 457-464.

53. Yamamoto J, Takayama T, Kosuge T, et al. - An isolated caudate lobectomy by the transhepatic approach for hepatocellular carcinoma in cirrhotic liver. Surgery 1992; 111: 699-702.

54. Couinaud C - Surgical anatomy of the liver revisited. Paris: Maugein \& Cie, 1989.

55. Elias D, Lasser PH, Desruennes E, et al. Surgical approach to segment I for malignant tumors of the liver. Surg Gynecol Obstet 1992; 175: 17-24.

Endereço para correspondência:

Dr. Fábio Luiz Waechter

Rua Barros Cassal 475/802

90035-030 - Porto Alegre - RS

Fax: (51) 3113310

E-mail:waechter@zaz.com.br 